\title{
23
}

\section{FEC and Multi-layer Video Coding For ATM Networks}

\author{
M.J. Riley and I.E.G. Richardson \\ The Robert Gordon University, Aberdeen AB9 1FR, Scotland
}

\begin{abstract}
Significant advances have been made in recent years on standards [1][2][3] for video compression. Compressed video data resulting from these coding standards is however susceptible to quality degradation when cell losses occur during transmission over an ATM network.

In [4][5] we have shown that multi-layer video coding using the Progressive Spectral Selection (PSS) mode of the JPEG algorithm produces parallel traffic streams (or scans) which show different tolerances to cell loss. Therefore, we apply varying quantities of FEC to each of the video traffic scans. The scans which are least sensitive to cell loss are transmitted with little or no error correction whereas the highly sensitive DC scan is heavily forward error corrected. A real video sequence is transmitted through a simulated ATM network before reanimation and subjective quality evaluation. We compare the video quality with that resulting from FEC on a baseline JPEG CODEC.

We conclude that the quality degradations introduced by cell loss have different effects on the two coding schemes. The base-line JPEG scheme with FEC provides excellent picture quality until the FEC fails where-upon a severely degraded frame results. The PSS JPEG scheme provides much higher FEC protection to the critical scan 0 data but less FEC to the other scans. As the cell error rate increases this will result in a more gradual overall deterioration in picture quality. In some applications the characteristics of the multi-layer scheme may be more desirable.
\end{abstract}

Keyword Codes: I4.2, C2.1, C2.2

Keywords: Compression (Coding), Network Architectures and Design, Network Protocols

\section{INTRODUCTION}

The International Telecommunications Union (ITU) have proposed that future public Broadband Integrated Service Digital Networks (B-ISDN) use the Asynchronous Transfer Mode (ATM) of operation. ATM networks transfer data in a continuous sequence of small fixed size cells. Cells from separate links are statistically multiplexed together over common links within the network. When traffic peaks coincide the capacity of the common link is exceeded resulting in congestion which if maintained for a sufficient length of time causes buffers to overflow and cells to be dropped. The majority of cell losses within ATM networks are attributable to buffer overflows and therefore cell loss occurs in bursts during times of congestion. The probability of cell loss therefore depends on the dimensioning of the network and the network management strategies adopted. An inverse relationship exists between 
network utilisation and cell loss rate.

ATM networks carry video traffic for applications such as video-on-demand and video conferencing. Recently a number of International Standards [1][2][3] have been produced for coding video into a bandwidth of a few Mbit/s or less. However, these coding standards do not address the problems of video transmission through a packet switched network. A particular problem is that data losses in compressed video traffic resulting from cell losses cause significant quality reductions in the decompressed video.

Existing transport protocols such as Transmission Control Protocol TCP [6] or ISO OSI TP [7] control packet loss using Automatic Repeat reQuest (ARQ) techniques. When real-time traffic such as video is sent through networks with a high bandwidth-delay product such as a B-ISDN ATM network, ARQ is no longer a suitable technique for error control. In [8] Forward Error Correction (FEC) has been proposed as a more suitable method of error control for coded video traffic over ATM networks.

Errors in ATM networks are due mainly to cell loss resulting from buffer overflow when statistically multiplexing several separate variable bit rate traffic streams onto a common channel. These losses are bursty with the peak cell loss rate being much higher than the average. Using FEC to protect against these cell error bursts may introduce a considerable bandwidth overhead.

\section{MULTI-LAYER VIDEO CODING}

The majority of video CODECs in existence code video into one continuous stream of compressed data. Some video coding schemes [9][10] however, have been proposed for coding video into a number of parallel data scans where each scan represents a different component of the coded data. Multi-layer coding schemes provide the opportunity for a communication system to treat traffic from each scan differently. One such multi-layer coding scheme is the PSS mode of the JPEG International Standard [1] (figure 2).

The JPEG coding standard implements still image compression. That is, each frame of the video sequence is treated independently. The image is split into $8 \times 8$ pixel blocks. Each block is transformed into the spatial frequency domain using the Discrete Cosine Transform (DCT) producing 64 coefficients numbered 0 to 63 . Figure 1 shows the basis images corresponding to each DCT coefficient. The coefficient at the lower left corner represents a DC component or uniform pixel block component. Spatial frequencies increase with distance from this lower left corner. The coefficients are then scanned in the order of increasing spatial frequency (zigzag order) and quantised according to a chosen quantisation table. The base-line sequential mode then specifies that all 64 coefficients are entropy encoded, whereas the PSS mode specifies that the coefficients are split into a number of separate scans as shown in figure 2 each representing a band of spatial frequencies and each scan is independently entropy encoded. The entropy encoded data is represented by a sequence of variable length codes.

In [4][5] we investigated the tolerances of each of these separate scans to data loss resulting from cell losses in an ATM network. We found that the different scans showed a large variation in tolerance to cell loss. Losses in the DC and low spatial frequency bands caused considerable quality loss whereas losses in the higher spatial frequency bands were much less objectionable. The scans $0 . .3$ were produced by splitting the DCT coefficients into spatial frequency bands consisting of DCT coefficients 0 (DC component), 1-5, 6-9 and 10-63 respectively. 


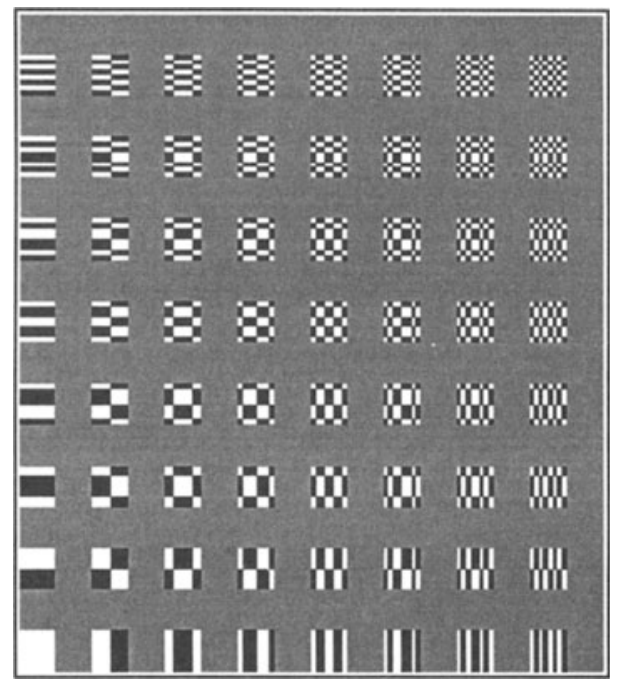

Figure 1: DCT Coefficients

\section{FORWARD ERROR CORRECTION (FEC)}

We adopt the FEC scheme used in [8] and based on a Reed Solomon code called RSE and described further in [11]. Blocks of $k$ cells are grouped together with $h$ redundant cells to form a FEC Block (FECB). The $h$ redundant cells contain FEC parity information and are calculated by striping across the FECB. Each stripe contains one $m$-bit symbol in an identical position within each cell and together form an RSE codeword. The codewords consist of $k+h$ symbols which are the coefficients of a polynomial $C(x)$ of degree $k+h$ with coefficients that are elements in the Galois field $\mathrm{GF}\left(2^{\mathrm{m}}\right)$. RSE is a systematic code; that is the first $k$ cells may be transmitted unchanged with the original data. Encoding and decoding are identical operations and involve solving the following simultaneous equations with $h$ unknowns:

$$
\left[\begin{array}{ccc}
a^{1(k+h-1)} & \ldots & a^{(1(0))} \\
a^{2(k+h-1)} & \ldots & a^{(2(0))} \\
\vdots & \ldots & \vdots \\
a^{h(k+h-1)} & \ldots & a^{(h(0))}
\end{array}\right]\left[\begin{array}{c}
c_{k+h-1} \\
\vdots \\
c_{0}
\end{array}\right]=\left[\begin{array}{lll}
0 & \cdots & 0
\end{array}\right]
$$

$a$ is a primitive element in $\mathrm{GF}\left(2^{\mathrm{m}}\right)$ and $c_{x}$ is a coefficient of $C(x)$. The code has the property of being able to recover all $k+h$ cells from any $k$ within each FECB providing that it is known which cells are lost. Lost cells may be identified by the inclusion of sequence numbers as specified for example in ATM Adaption Layer 1 (AAL1) [12]. 


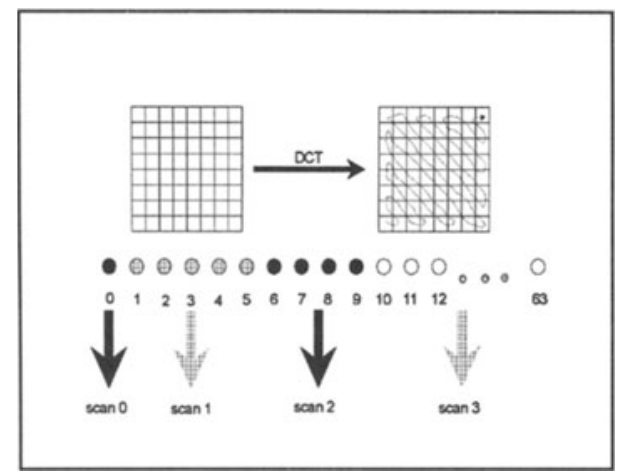

Figure 2: PSS Mode of JPEG

We simulated (using Monte-Carlo techniques) the performance of RSE for $k=50$ and with various values of $h$ for transmission over an ATM network during a period of congestion. We assumed that cell losses were independent and that the burst cell loss rate is 1 in 20 . The results of this simulation are shown in figure 3 and can be confirmed by calculation.

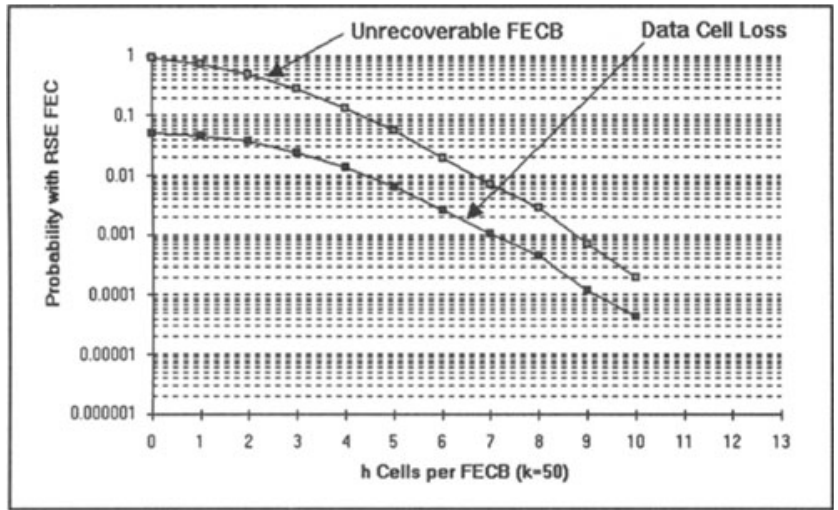

Figure 3: RSE FEC ( 1 in 20 cell loss)

The effective data cell loss rate and the probability that an FECB cannot be corrected is shown. It should be noted that remaining cell losses are concentrated into bursts of loss within FECBs which could not be corrected.

\section{SIMULATION TEST-BED}

At the Robert Gordon University we have constructed a simulation test-bed which allows us to simulate the transmission of a real sequence of coded video through a communication network and then re-animate the sequence for subjective quality evaluation.

This test-bed consists of a laser video disk player/recorder under the automatic control of a Personal Computer (PC). The laser video disk player/recorder stores a sequence of video as 
consecutive individually addressable frames on an analogue disk. Software has been written for the PC which causes successive video frames to be accessed, then digitised and converted to a sequence of graphics images in the PC. These digitised images are then coded using a software CODEC, and passed through a simulated network. The received data is decoded and then re-animated onto the laser video disk player/recorder. The re-animated sequence can be viewed in real-time and subjectively evaluated.

We have implemented the software to control frame capture/animation, coding/decoding, and network simulation at the Robert Gordon University in $\mathrm{C}$ and $\mathrm{C}++$.

\section{EXPERIMENT}

We performed an experiment using our simulation test-bed to determine whether using FEC in conjunction with multi-layer coded video offered bandwidth and quality advantages over using FEC in conjunction with uni-layer coded video when transmitting through an ATM network which introduces high burst levels of cell loss.

We considered two video sequences:-

(1) An 8 second uni-layer coded video sequence using the base-line JPEG CODEC. This data stream was sequentially packaged into the payload ( 47 bytes per cell +1 byte sequence number) of a sequence of ATM cells and then further encoded using RSE prior to transmission through a simulated ATM network. 7 redundant cells were added to each group of 50 data cells to form FECBs.

(2) An 8 second multi-layer coded video sequence using the PSS JPEG CODEC. This produced four parallel streams of video data using the same DCT quantisation table as in the uni-layer case. These four video streams (scans) were produced from grouping the DCT coefficients numbered 0 into scan $0,1-5$ into scan 1, 6-10 into scan 2 and 11-63 into scan 3. Traffic resulting from each scan was sequentially packaged into the payload ( 47 bytes per cell +1 byte sequence number) of a sequence of ATM cells. Cells were grouped into FEC Blocks (FECB) which consisted of 50 data cells plus a number $h$ of redundant cells formed using RSE encoding. The number $h$ was varied for each scan so that scans highly sensitive to cell loss carried most FEC overhead and scans least sensitive to cell loss carried least or no FEC overhead. The values of $h$ chosen for scans $0,1,2$ and 3 are 10, 4, 4 and 0 respectively.

Overall, the PSS mode is not as efficient as the base-line mode due to the additional overheads of multi-layer coding. Therefore, we chose to add correspondingly less FEC to the multi-layer coding scheme such that the overall bandwidth required for the two coding schemes is identical.

These two video sequences were transmitted through our ATM network simulation. We assumed that for the full 8 second simulation time the ATM network is operating under conditions of congestion and therefore burst cell loss conditions. We assumed a burst cell loss rate of 1 in 20 where cell loss is independent within the burst.

\section{RESULTS}

The traffic patterns resulting from video streams (1) and (2) are illustrated in figures 4 and 5 respectively which show frame numbers $(1130 . .1330 ; 200$ frames in all) versus cells per frame time (1/25s). These graphs include the additional traffic due to redundant FEC information. As intended, the average data rate in each case is approximately identical. 


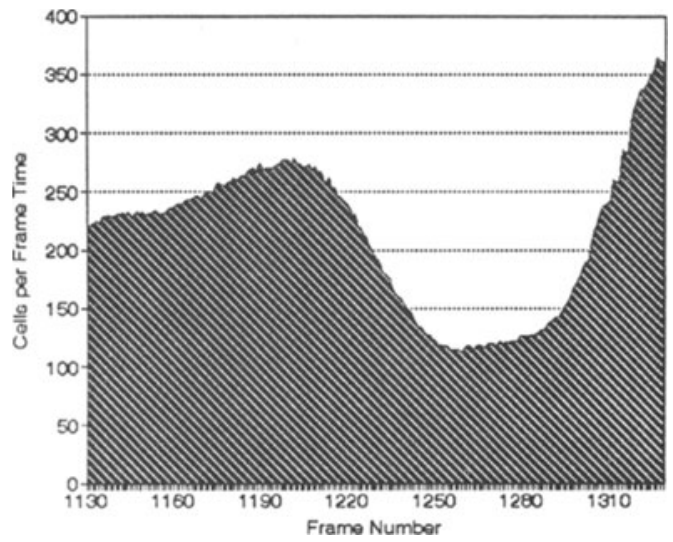

Figure 4: Baseline JPEG with FEC

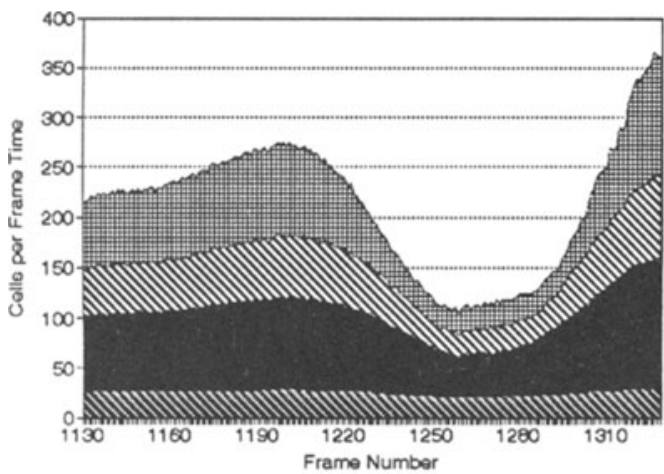

MW $\operatorname{Scan} 0 \mathrm{Scan} 1 \mathrm{MW} \operatorname{Scan} 2$ 페요 $\operatorname{Scan} 3$

Figure 5: PSS JPEG with FEC

We evaluated the received re-animated video sequences subjectively by viewing them in real-time. Sequence (1) showed periodic errors approximately every 1.5 seconds which were highly objectionable. An example of a frame with errors from sequence 1 is shown in figure 6 . Sequence (2) showed no highly objectionable errors (errors affecting scan 0 ) during the simulation sequence. The remaining errors in sequence (2) produce some quality degradation but no dramatic collapse in picture quality. An example of some frames from sequence 2 with errors in various scans are shown in figures 7 and 8 . Figure 7 shows some distortion resulting from scan 1 errors in the top right hand corner. Both figures 7 and 8 show distortion from errors in scans 2 and 3 around the edges of the helicopter. 


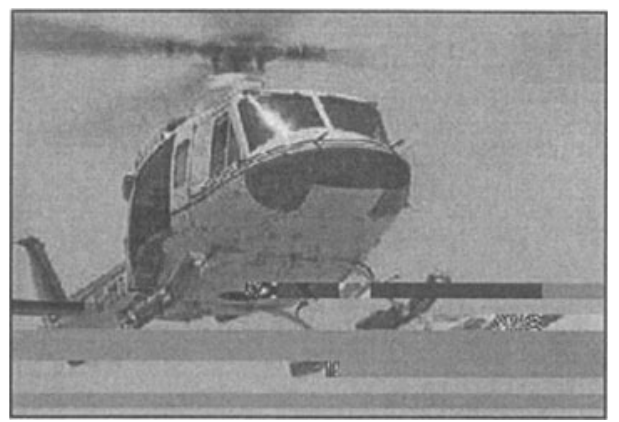

Figure 6: Frame with errors using Baseline JPEG

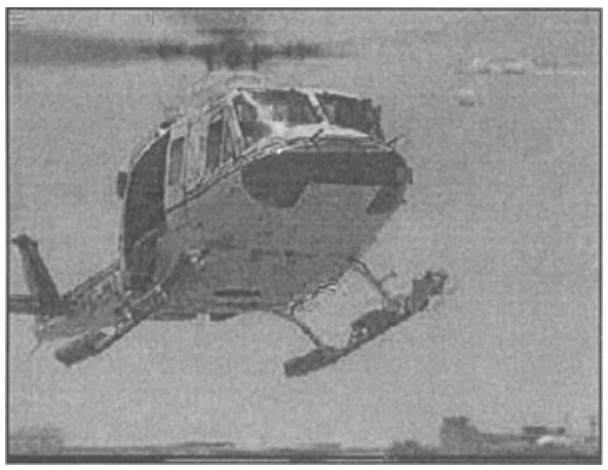

Figure 7: Frame with errors (PSS JPEG)

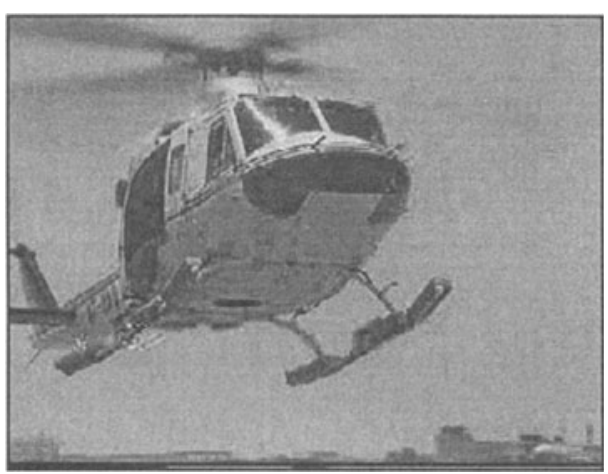

Figure 8: Frame with errors (PSS JPEG) 


\section{DISCUSSION}

Any cell losses which pass uncorrected in the base-line scheme cause distortions which propagate across the image and are highly objectionable. Only cell losses which pass uncorrected in scan 0 of the multi-layer scheme cause these highly objectionable distortions. Cell losses in the other scans produce distortions which decrease in severity with spatial frequency and are less likely to propagate across the image. Since scan 0 is protected with 10 redundant cells per FECB ( 50 data cells plus 10 parity cells) it can be estimated from figure 3 that the probability of an unrecoverable FECB using RSE is 0.0002 . From figure 5 it can be seen that the scan 0 data rate is approximately 25 cells (or $25 / 60$ FECBs) per frame (1/25 second). Therefore, we estimate that on average an unrecoverable FECB in scan 0 would occur once every 12195 frames (approximately every 8 minutes) under the conditions of our experiment. Each unrecoverable FECB would then result in a number of lost cells. However, errors in the higher frequency scans, resulting in less severe distortion, occur much more frequently. This illustrates that using multi-layer coding may produce a system which in some ways is more resilient to variations in the network QOS. Further work is required to optimise the allocation of FEC cells to the various scans in the multi-layer scheme for a particular application.

\section{CONCLUSIONS}

FEC using RSE is an effective error control technique for ATM networks which may be subject to periods of burst cell loss resulting from buffer overflow. Using FEC in conjunction with multi-layer video coding using the PSS mode of JPEG provides the opportunity to reduce the levels of FEC applied to some of the scans. The multi-layer scheme provides the opportunity to greatly reduce the probability of highly objectionable (scan 0 ) errors. This is balanced by increased error activity in the other scans causing some overall quality degradations. The multi-layer scheme may offer the potential for building a system which is adaptable to a wider range of network QOS. The nature of the quality degradation changes between the two schemes. The relative merits of the two schemes for a specific application requires further work involving careful subjective quality evaluation.

\section{REFERENCES}

1. "Digital compression and coding of continuous-tone still images", ISO 10918.

2. "Coding of moving pictures and associated audio for digital storage media at up to about 1.5 Mbit/s", ISO 11172.

3. "CODEC for audio-visual services at $\mathrm{p} x$ 64Kbit/s", CCITT Recommendation H.261.

4. I.E.G. Richardson, M.J. Riley, "ATM cell loss effects on video traffic", IEE Colloquium on Multi-media Communication Systems, March 1994.

5. I.E.G. Richardson, M.J. Riley, "ATM cell loss effects on a Progressive JPEG Video CODEC", Accepted for presentation at Third International Conference on Broadband Islands, June 1994.

6. Postel, Transmission Control Protocol, RFC 793.

7. ISO OSI Transport Protocol, ISO 8073.

8. E.W. Biersack, "Performance Evaluation of Forward Error Correction in an ATM Environment", IEEE Journal on Selected Areas in Communications, Vol. 11, No.4, May 1993.

9. M. Ghanbari, "An Adapted H.261 two layer video codec for ATM Networks", IEEE Trans. on Communications, Vol.40,No.9, September 1992, pp1481-1490.

10. C. Guillemot, R.Ansari, "Layered Coding Schemes for Video Transmission on ATM Networks", Journal of Visual Communication and Image Representation, Vol.5,No.1,pp62-74, 1994.

11. "High Performance Networks: Frontiers and Experience", Kluwer Academic Publishers, Edited

A. Tantawy, pp161-163.

12. ITU:Recommendation I.363. "B-ISDN ATM Adaption Layer (AAL) Specification", Geneva 1991. 